\title{
Increased Oral Bioavailability of Piperine from an Optimized Piper nigrum Nanosuspension
}

\author{
Authors \\ Fatiqa Zafar ${ }^{1}$, Nazish Jahan ${ }^{1}$, Khalil-Ur-Rahman ${ }^{2}$, Haq Nawaz Bhatti ${ }^{1}$
}

Affiliations

1 Department of Chemistry, University of Agriculture, Faisalabad, Pakistan

2 Department of Biochemistry, University of Agriculture, Faisalabad, Pakistan

Key words

nanosuspension, bioavailability, piperine, pharmacokinetics, Piper nigrum, Piperaceae

received May 11, 2018

revised September 29, 2018

accepted October 9, 2018

Bibliography

DOI https://doi.org/10.1055/a-0759-2208

Published online October 24, 2018 | Planta Med 2019; 85:

249-257 @ Georg Thieme Verlag KG Stuttgart · New York I ISSN 0032-0943

Correspondence

Fatiqa Zafar, PhD Scholar

Natural Products Lab, Department of Chemistry, Faculty of Sciences, University of Agriculture

Jail Road, Faisalabad (38000), Punjab, Pakistan

Phone: + 92419200161 , Fax: + 92419200764

fatiqazafar@gmail.com

Supporting information available online at

http://www.thieme-connect.de/products

\section{ABSTRACT}

The aim of the present study was to enhance the pharmaceutical potential and oral bioavailability of piperine, which is the bioactive constituent of Piper nigrum, using the nanosuspension approach. Nanoprecipitation, which is a simple and reproducible process, was used for nanosuspension formulation. To prepare a pharmaceutical-grade nanosuspension with the required particle size, important formulation parameters (amount of plant extract, concentration of stabilizer, and antisolvent-to-solvent ratio) were optimized using the central composite design of response surface methodology. The optimized nanosuspension was characterized using scanning electron microscopy, atomic force microscopy, Fourier transform infrared spectroscopy, and in vitro dissolution testing as well as by measuring the zeta potential. In vivo pharmacokinetic studies were conducted to determine the bioavailability of the prepared nanosuspension. Results of the optimization study indicated that $0.13 \%$ plant extract, $0.25 \%$ stabilizer, and an antisolvent-to-solvent ratio of 10.0 were the best parameters to obtain a homogeneous nanosuspension with the required particle size. The optimized nanosuspension demonstrated a mean particle size, polydispersity index, and zeta potential of $172.5 \mathrm{~nm}, 0.241$, and $-16.6 \mathrm{mV}$, respectively. The results of the characterization studies illustrated that the nanosuspension was in the nanometer size range and had good surface morphology. The optimized nanosuspension showed a better dissolution rate and a 3.65-fold higher oral bioavailability for the $P$. nigrum nanosuspension than its coarse suspension. The present outcomes clearly demonstrated that to obtain an effective therapeutic potential, nanoformulation of medicinal plants is a better alternative than conventional dosage forms.

\section{Introduction}

Piper nigrum L. (Piperaceae), which is generally known as black pepper, is extensively used in household spices and has paramount importance in the field of alternative medicine [1]. Piperine is the major alkaloid of $P$. nigrum and possesses diverse pharmacological activities [2] such as antihypertensive [3], antioxidant [4], hypolipidemic [5], antiasthmatic [6], hepatoprotective [7], and antimicrobial [8] properties. It is not only a bioavailability enhancer but also a potent reactive oxygen species quencher and it can inhibit lipid peroxidation [9-11]. Being a bioavailability enhancer, piperine serves as a drug receptor and potentiates the drug via conformational interactions by making target cells more receptive [2].

Although piperine acts as a bioavailability enhancer, its water solubility is negligible [12]. Its poor water solubility results in its limited bioavailability in biological systems and necessitates the use of a higher dose of drugs to obtain the anticipated pharmacological response. Therefore, it is imperative to improve the solubility of piperine to obtain its maximum therapeutic efficacy in the 


$\begin{array}{ll}\text { ABBREVIATIONS } \\ \text { 3D } & \text { three-dimensional } \\ \text { AFM } & \text { atomic force microscopy } \\ \text { ANOVA } & \text { analysis of variance } \\ \text { AS/S ratio } & \text { antisolvent-to-solvent ratio } \\ \text { AUC } & \text { area under the curve } \\ \text { CCD } & \text { central composite design } \\ \text { CV } & \text { coefficient of variation } \\ \text { DLS } & \text { dynamic light scattering } \\ \text { FTIR } & \text { Fourier transform infrared spectroscopy } \\ \text { HPMC } & \text { hydroxypropyl methylcellulose } \\ \text { PDI } & \text { polydispersity index } \\ \text { RSM } & \text { response surface methodology } \\ \text { SEM } & \text { scanning electron microscopy }\end{array}$

biological system. Among the various approaches, nanosuspension formulation is the method of choice that enhances the delivery of sparingly water-soluble drugs $[13,14]$.

Nanoformulated herbal drugs possess efficient biopharmaceutical properties and desirable target characteristics [15]. These nano-sized phytotherapeutic agents offer better pharmaceutical benefits over traditional herbal preparations, including enhanced solubility, improved bioavailability, reduced medicinal doses, and better residence time of drugs in biological systems [16]. The enhanced bioavailability due to the smaller size and greater surface area [17] consequently reduces the treatment dose [18]. Nanosuspensions can also improve the pharmacokinetics of pharmaceutics [19].

To obtain an effective and pharmaceutically stable nanosuspension with the required particle size and desired morphology, it is imperative to optimize formulation parameters. The optimization of each parameter is time-consuming and expensive and does not determine the effect of individual parameters on various responses [20]. Moreover, large numbers of trials are required in factorial design, which eventually does not provide the required information (interactive effect), making the situation more complex [21]. To overcome these problems, optimization studies can be effectively conducted using RSM [22].

The present research was conducted to enhance the oral bioavailability of $P$. nigrum plant extract by formulating its nanosuspension. Different process parameters were optimized using RSM to obtain a homogenous nanosuspension with a minimum particle size. The optimized nanosuspension was characterized by spectroscopic techniques and evaluated by in vitro dissolution testing and in vivo pharmacokinetic studies.

\section{Results and Discussion}

Nanosuspensions were prepared by the antisolvent precipitation method using an HPMC stabilizer. The formulation parameters considered were the amount of plant extract $(A)$, concentration of stabilizer (B), and AS/S ratio (C). These parameters were optimized using the CCD of RSM to obtain a homogenous nanosuspension with a minimum particle size (z-average; $\mathrm{nm}$ ) and stan- dard PDI value. The statistical experimental design suggested that the quadratic model was the most suitable model, with the smallest $p$ values and largest Fisher values (F values), to explain the relationship between independent variables (amount of plant extract, concentration of stabilizer, and AS/S ratio) and response variables [particle size (R1) and PDI (R2)]. After selecting the model, regression equations (Equations 1 and 2 ) for the response variables were established. The positive sign before the coefficients suggests a synergistic effect of independent variables on particle size and PDI reduction, whereas the negative sign represents an antagonistic effect.

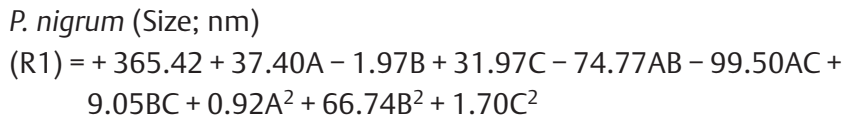

(Equation 1)

P. nigrum (PDI)

$$
\begin{aligned}
(R 2)= & +0.42-0.012 A-0.047 B+0.060 C-0.16 A B+0.049 A C- \\
& 0.071 B C-4.633 E-003 A^{2}+0.15 B^{2}-0.080 C^{2}
\end{aligned}
$$

(Equation 2)

ANOVA was used to evaluate the linear, quadratic, and interactive effects of independent variables on R1 and R2. The probability $(p=0.008$ and $p<0.0001)$ and $F(9.42$ and 48.82$)$ values for $R 1$ and $R 2$, respectively, reflected the significance of the quadratic model for the optimal production of nanosuspensions with the desired properties ( $\vee$ Tables 1 and 2 ). P values were used as a tool to check the significance of the model terms. A $p$ value of $<0.0500$ indicated that the model terms were significant. The smaller the $p$ value, the more significant the corresponding coefficient was. The variables $A$ and $C$ and the interactions $A B$, $A C$, and $B^{2}$ had a significant effect on the nanosizing of $P$. nigrum, and the parameters $B, C, A B, A C, B C, B^{2}$, and $C^{2}$ had a significant effect on reducing $P D I$; the remaining parameters showed no effect or an inverse relationship on particle size and PDI reduction ( $\triangleright$ Tables 1 and 2 ).

The nonsignificant lack of fit F value of R1 (0.47) and R2 (1.47) demonstrated good predictability of the model. The quality of fit for the quadratic model was further evaluated using the coefficient of determination $\left(R^{2}\right)$. $R^{2}$ for particle size $(0.8945)$ and PDI (0.9777) indicated 89.45 and $97.77 \%$ variability in both responses. The model was stronger and predicted a better response as $\mathrm{R}^{2}$ was closer to 1.000 . In previous studies, a regression model with $\mathrm{R}^{2}>0.9000$ has been considered to have a very high correlation [23]. The CV was calculated to be 12.41 and $9.26 \%$ for R1 and R2, respectively, and was found to be satisfactory ( $\vee$ Tables $\mathbf{1}$ and $\mathbf{2}$ ).

The influence of all independent variables on the various responses of the nanosuspension formulation of $P$. nigrum was evaluated using 3D response surface plots. In each plot, the combined effect of two variables was simultaneously examined, whereas a third factor was kept at its central point. Response surface plots showing the interactive effect of all independent variables on the particle size and PDI of $P$. nigrum nanosuspensions are presented in $\mathbf{F i g s .} \mathbf{1}$ and $\mathbf{2}$. These plots show that all three formulation parameters have a significant impact on particle size and PDI reduction of P. nigrum nanosuspensions. However, the impact of the 
- Table 1 ANOVA for response surface quadratic model for the particle size of $P$. nigrum nanosuspensions.

\begin{tabular}{|c|c|c|c|c|c|c|}
\hline Source of variance & Sum of square & df & Mean square & F value & $P$ value Prob $>F$ & Remarks \\
\hline Model & $2.23 \mathrm{E}+05$ & 9 & 24740.20 & 9.42 & 0.0008 & Significant \\
\hline A-Plant extract & 19100.72 & 1 & 19100.72 & 7.28 & 0.0224 & \\
\hline B-Stabilizer concentration & 53.04 & 1 & 53.04 & 0.02 & 0.8898 & \\
\hline C-Antisolvent/solvent ratio & 13956.52 & 1 & 13956.52 & 5.32 & 0.0438 & \\
\hline$A B$ & 44727.41 & 1 & 44727.41 & 17.04 & 0.0021 & \\
\hline$A C$ & 79209.96 & 1 & 79209.96 & 30.17 & 0.0003 & \\
\hline BC & 654.86 & 1 & 654.86 & 0.25 & 0.6283 & \\
\hline$A^{2}$ & 12.24 & 1 & 12.24 & $4.66 \mathrm{E}-03$ & 0.9469 & \\
\hline $\mathrm{B}^{2}$ & 64199.45 & 1 & 64199.45 & 24.45 & 0.0006 & \\
\hline$C^{2}$ & 41.53 & 1 & 41.53 & 0.016 & 0.9024 & \\
\hline Residual & 26252.41 & 10 & 2625.24 & & & \\
\hline Lack of fit & 8403.54 & 5 & 1680.71 & 0.47 & 0.7860 & Nonsignificant \\
\hline Pure error & 17848.87 & 5 & 3569.77 & & & \\
\hline Cor total & $2.49 \mathrm{E}+05$ & 19 & & & & \\
\hline $\mathrm{R}^{2}$ & 0.8945 & & & $\operatorname{Adj} R^{2}$ & 0.7996 & \\
\hline Pred $R^{2}$ & 0.6395 & & & CV (\%) & 12.41 & \\
\hline Adeq precision & 11.685 & & & & & \\
\hline
\end{tabular}

- Table 2 ANOVA for response surface quadratic model for PDI of $P$. nigrum nanosuspensions.

\begin{tabular}{|c|c|c|c|c|c|c|}
\hline Source of variance & Sum of square & df & Mean square & F value & $P$ value Prob $>F$ & Remarks \\
\hline Model & 0.82 & 9 & 0.091 & 48.82 & $<0.0001$ & Significant \\
\hline A-Plant extract & $1.93 \mathrm{E}-03$ & 1 & $1.93 \mathrm{E}-03$ & 1.04 & 0.3323 & \\
\hline B-Stabilizer concentration & 0.03 & 1 & 0.030 & 16.34 & 0.0024 & \\
\hline C-Antisolvent/solvent ratio & 0.05 & 1 & 0.050 & 26.80 & 0.0004 & \\
\hline$A B$ & 0.21 & 1 & 0.210 & 115.17 & $<0.0001$ & \\
\hline AC & 0.019 & 1 & 0.019 & 10.28 & 0.0094 & \\
\hline BC & 0.04 & 1 & 0.040 & 21.76 & 0.0009 & \\
\hline$A^{2}$ & 3.09E-04 & 1 & 3.09E-04 & 0.17 & 0.692 & \\
\hline$B^{2}$ & 0.33 & 1 & 0.330 & 176.53 & $<0.0001$ & \\
\hline$C^{2}$ & 0.092 & 1 & 0.092 & 49.52 & $<0.0001$ & \\
\hline Residual & 0.019 & 10 & $1.86 \mathrm{E}-03$ & & & \\
\hline Lack of fit & 0.011 & 5 & $2.21 \mathrm{E}-03$ & 1.47 & 0.3416 & Nonsignificant \\
\hline Pure error & $7.53 \mathrm{E}-03$ & 5 & $1.51 \mathrm{E}-03$ & & & \\
\hline Cor total & 0.84 & 19 & & & & \\
\hline$R^{2}$ & 0.9777 & & & Adj $R^{2}$ & 0.9577 & \\
\hline Pred $R^{2}$ & 0.8762 & & & CV (\%) & 9.26 & \\
\hline Adeq precision & 27.348 & & & & & \\
\hline
\end{tabular}

amount of plant extract is more prominent. Minimum particle size and PDI are observed when the amount of plant extract is less. The classical crystallization theory explains the impact of drug concentration (plant extract in the present case) on particle size. According to this theory, during nanoformulation, the precipitation of nanoparticles involves a series of steps including nuclea- 

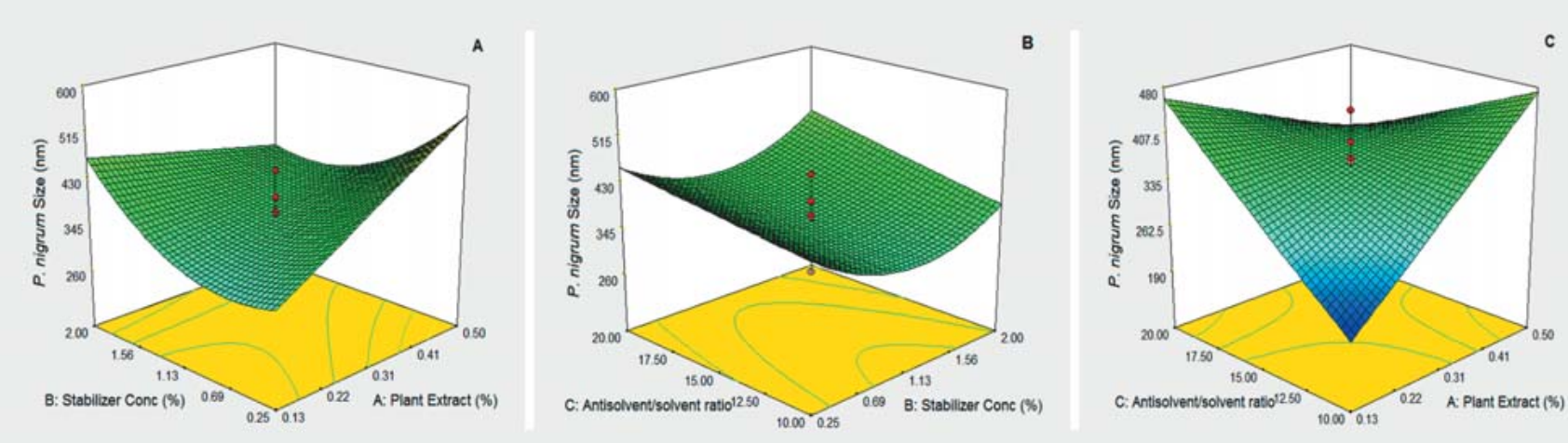

- Fig. 1 3D response surface graphs for the particle size of $P$. nigrum nanosuspensions. (A) Amount of plant and concentration of stabilizer,

(B) concentration of stabilizer and AS/S ratio, and (C) amount of plant and AS/S ratio.
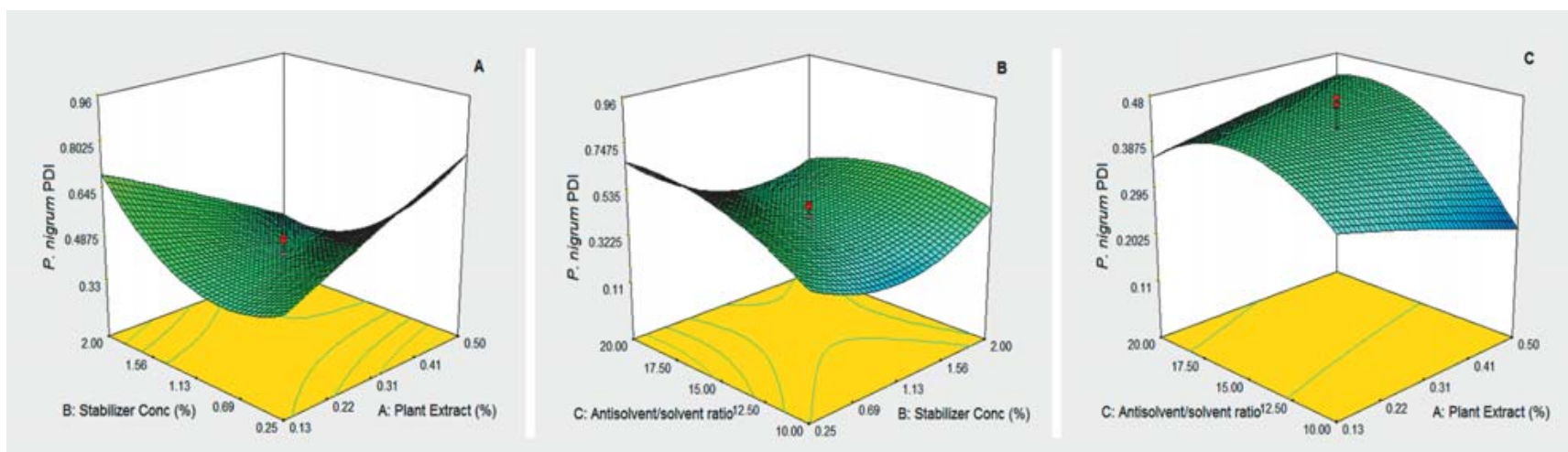

- Fig. 2 3D response surface graphs for PDI of P. nigrum nanosuspensions. (A) Amount of plant and concentration of stabilizer, (B) concentration of stabilizer and AS/S ratio, and (C) amount of plant and AS/S ratio.

tion, molecular growth, and growth by coagulation and condensation, followed by agglomeration. Furthermore, the rate of each step governs the final particle size and particle size distribution. Supersaturation is the crucial driving force of this process and determines not only the nucleation rate but also the diffusioncontrolled growth rate. The nucleation and growth of particles occur simultaneously, and both compete for supersaturation [24]. At a higher drug concentration, due to higher supersaturation, the rapid diffusion-controlled growth and agglomeration rates were more dominant than the nucleation rate that gave rise to larger particles $[25,26]$.

A gradual increase in particle size was also observed by increasing the concentration of HPMC. This may be due to the fact that an excessive amount of polymer increases particle size by increasing the size of the outer polymer surface and inhibits diffusion between the solvent and antisolvent during precipitation. Moreover, an increase in osmotic pressure by increasing polymer concentration results in an enhanced attraction among colloidal particles, thus leading to a larger particle size [27]. Furthermore, an increase in particle size and PDI by increasing the concentration of the stabilizer may result from Ostwald ripening [28]. In another study, the particle size of resveratrol nanosuspension was increased by increasing the amount of the drug and concentration of the stabilizer [26].

Desirability and overlay plots created using Design Expert Software (version 7.1, Stat-Ease, Inc.) to attain the optimum values of independent variables (A, B, and $C$ ) for the formulation of $P$. nigrum nanosuspension with a minimum particle size and PDI are shown in > Fig. 3 . These plots further confirm the validity of the selected quadratic model of CCD of RSM in optimization studies. Particle size $(172.5 \mathrm{~nm})$, PDI $(0.241)$, and zeta potential $(-16.6 \mathrm{mV})$ confirmed the stability of the formulated nanosuspension ( $\triangleright$ Fig. 4 A, B).

AFM of $P$. nigrum nanosuspension was conducted for illustrating the particle distribution. Thus, the overall particle distribution was observed to be uniform; however, the nanoparticles were observed to be submerged in the polymer layer at some places. The observed mean particle height of the $P$. nigrum nanosuspension was $55.78 \mathrm{~nm}$ ( $\triangleright$ Fig. 5). The particle size observed by AFM was smaller $(50.78 \mathrm{~nm})$ than that $(172.9 \mathrm{~nm})$ obtained by DLS. Fritzen-Garcia et al. [29] stated that the particle size of a nanosuspension measured by AFM was smaller than that measured by 

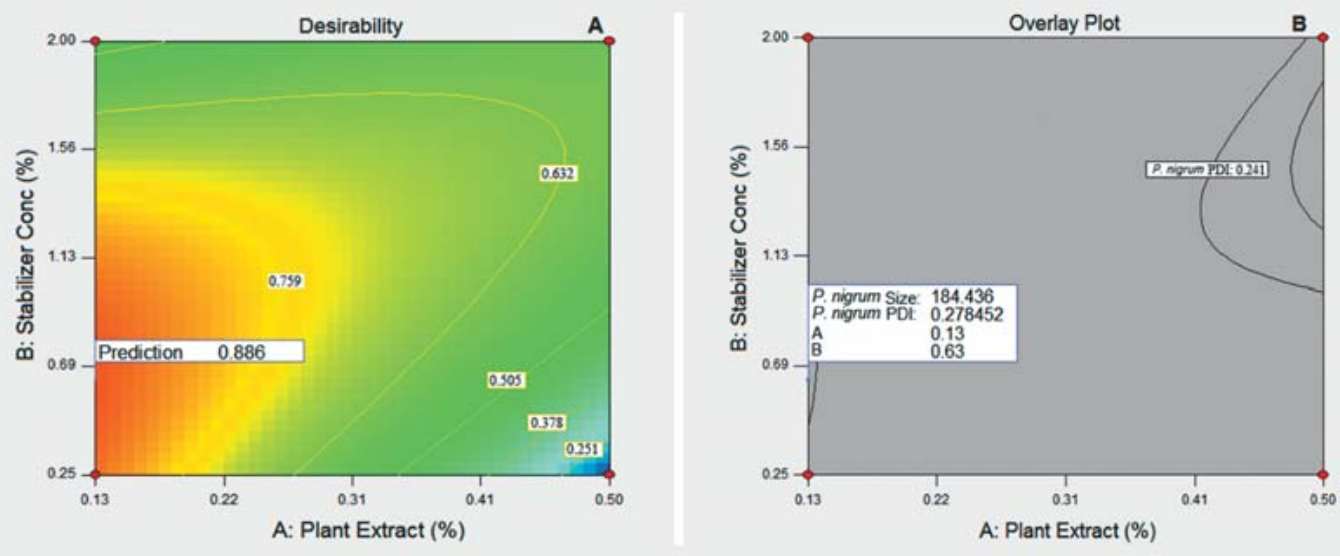

- Fig. 3 (A) Desirability and (B) overlay plot for particle size and PDI values of $P$. nigrum nanosuspensions.

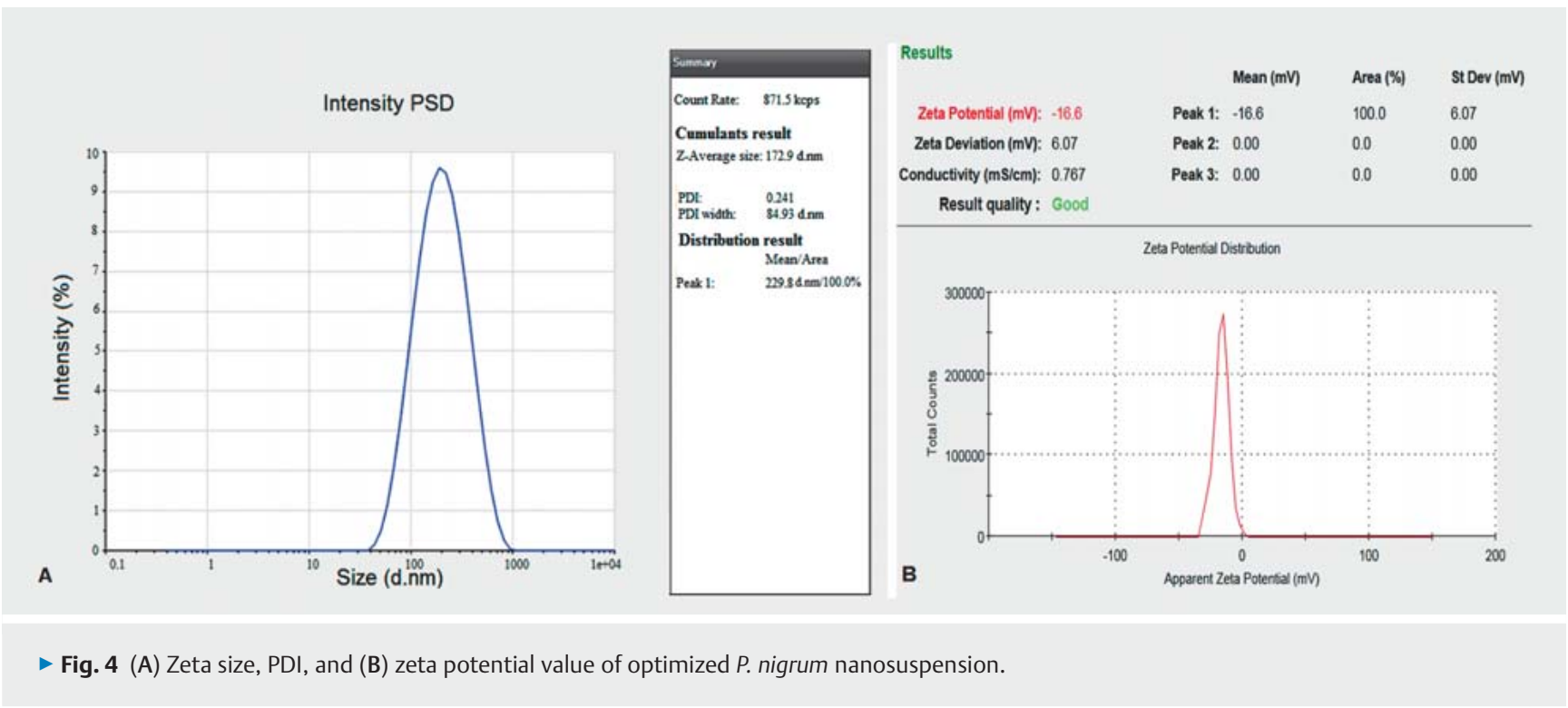

DLS. This is due to the presence of a solvent in measurements taken by DLS, which causes nanoparticle swelling [30].

SEM of the $P$. nigrum nanosuspension at two different resolutions demonstrated an average particle size of $<1 \mu \mathrm{m}$ ( $\triangleright$ Fig. 6), which indicated no aggregation of particles. HPMC provided better stability to the nanosuspension, even after lyophilization. The particles were discrete and uniform and had a spherical- and rodlike shape with a smooth surface, which is a quality characteristic of HPMC [31, 32].

Compared to the coarse plant extract, FTIR analysis of $P$. nigrum revealed a slight shift or disappearance of some peaks in the spectrum of the nanosuspension (Fig. 1S, Supporting information). However, the peak characteristics of the functional group region (3500-1900 $\mathrm{cm}^{-1}$ ) showed smaller variations than peaks in the fingerprint region $\left(1600-450 \mathrm{~cm}^{-1}\right)$. In the nanosuspension, peaks in the region of $3200-3550 \mathrm{~cm}^{-1}$, which were characteristics of an $-\mathrm{OH}$ bond, became more intense with a slight decrease in wavenumber. This may be due to $\mathrm{H}$-bonding [33]. The other peaks varied to a very small extent from $2936.77 \mathrm{~cm}^{-1}$ and $2855.42 \mathrm{~cm}^{-1}$ in the plant extract to $2917.63 \mathrm{~cm}^{-1}$ and $2850.21 \mathrm{~cm}^{-1}$ in the nanosuspension. These peaks $\left(2936.77 \mathrm{~cm}^{-1}\right.$ and $2855.42 \mathrm{~cm}^{-1}$ ) illustrated the presence of symmetric and asymmetric $-\mathrm{CH}$ and $-\mathrm{CH}_{2}$ stretching. The peak at $1632.15 \mathrm{~cm}^{-1}$ represented -CO-N stretching, and a sharp peak at $927.91 \mathrm{~cm}^{-1}$ was characteristic of - $\mathrm{CO}$ stretching. A more prominent peak at $1444.42 \mathrm{~cm}^{-1}$ was representative of $-\mathrm{CH}_{2}$ bending. The peaks at wavenumbers $803.80,846.49$, and $821.20 \mathrm{~cm}^{-1}$ were for out of the plane $-\mathrm{CH}$ bending and two adjacent substituted hydrogen atoms of 1,2,4-trisubstituted phenyl, respectively. All these peaks confirmed the presence of piperine as the major constituent in the coarse extract of $P$. nigrum. The fingerprint region $(1600-450 \mathrm{~cm}$ ${ }^{-1}$ ) of the spectrum of the nanosuspension showed greater resemblance with the spectrum of the stabilizer than with the spectrum of the coarse plant extract. This may be due to some physical in- 
teractions between the plant extract, stabilizer, and antisolvent [34] during nanoformulation. The present outcomes, which were similar to those obtained in a previous study [35], indicated that the plant extract in pure form or in nanosuspension form has the same structural features in terms of functional groups.

Results of the dissolution profile of the $P$. nigrum coarse extract and nanosuspension are presented in $\mathbf{F i g . ~ 7 . ~ A ~ g r e a t e r ~ c o n c e n - ~}$ tration of piperine in the dissolution medium was observed for the nanosuspension (73.66\%) after 120 min than in the coarse extract (14.37\%). This indicated a 3.65 -fold increase in the dissolution behavior of the nanosuspension. The dramatically enhanced dissolution rate of the nanosuspension may result from the increased effective surface area [36] and decreased particle size [31] in accordance with Noyes Whitney's equation [37]. Comparable results were found by Kakran et al. [38], who fabricated nanoparticles of silymarin, hesperetin, and glibenclamide by the evaporative precipitation of the nanosuspension and concluded that the dissolution rate increased by reducing the particle size and increasing the surface area available for dissolution.

In in vivo trials, the concentration-time graph in pharmacokinetic studies showed a higher concentration of piperine in the plasma samples of rats treated with the $P$. nigrum nanosuspension than in those of rats treated with the coarse suspension at all studied time intervals ( $\bullet \mathbf{F i g} \mathbf{8}$ ). The maximum concentration $\left(C_{\max }\right)$ of piperine was achieved after $1 \mathrm{~h}\left(\mathrm{~T}_{\max }\right)$ of oral administration of the $P$. nigrum nanosuspension; this maximum concentration was 1.73 -fold higher than that of the coarse suspension. After $T_{\max }$, the concentration of piperine started decreasing, indicating drug clearance from the biological system. However, the drug clearance rate was slower for the nanosuspension than for the coarse suspension, demonstrating its sustained release and greater residence time in the biological system. Tian et al. [39] found a prolonged residence time of the nanosuspension. The greater mean $A U C\left(A \cup C_{0-24 h}\right)$ for the nanosuspension than for the coarse suspension ( $\vee$ Table 3 ) represents a 2.7 -fold increase in the bioavailability of the $P$. nigrum nanosuspension compared to its coarse suspension. Significant improvement in the $C_{\max }$ and AUC of the nanosuspension indicated better in vivo exposure of the nanosuspension through particle size reduction [40], which can be explained by the improved saturation solubility of the nanoparticles, as they are absorbed without the initial time-consuming step [36]. Furthermore, nanosuspension preparation for oral administration results in effective therapeutic concentrations in the blood because solubility and absorption problems in the gastrointestinal tract can be managed by extensively reducing the particle size of the nanosuspension [41,42].

To the best of our knowledge, the formulation and pharmacokinetic evaluation of the nanosuspension of the $P$. nigrum plant extract have been conducted for the first time. In summary, nanosuspensions possess smaller particle sizes, higher surface areas, faster dissolution rates, less drug doses, lesser side effects, and enhanced bioavailability [43]. The results showed a distinctive comparison of the pharmacokinetics and dissolution properties between the nanosuspension and the coarse suspension of $P$. nigrum.

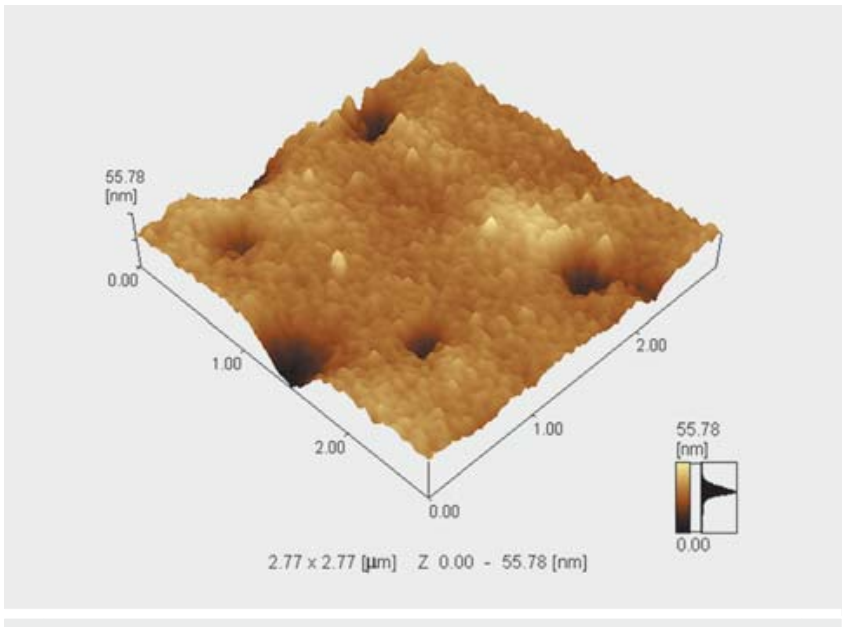

- Fig. 5 AFM image of $P$. nigrum nanosuspension.

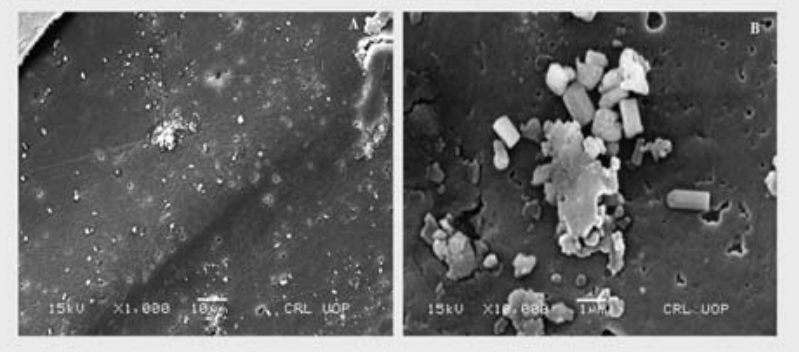

- Fig. 6 SEM images of $P$. nigrum nanosuspension at (A) $1000 \times$ magnification (10 $\mu \mathrm{m}$ bar size) (B) 10000× magnification ( $1 \mu \mathrm{m}$ bar size).

\section{Materials and Methods}

\section{Plant collection and extract preparation}

P. nigrum L. (fruit) was purchased from the local market of Faisalabad and identified by a plant taxonomist (Dr. Mansoor Hameed) at the Department of Botany, University of Agriculture, Faisalabad (UAF). A voucher specimen (228-2-2016) was deposited at the herbarium of the Department of Botany, UAF. The plant material was grounded to a fine powder after washing and drying. Fat/oil contents were removed with $n$-hexane $(1: 10 \mathrm{w} / \mathrm{v})$ using a Soxhlet extractor. To obtain crude piperine, defatted plant material $(30 \mathrm{~g})$ was extracted with ethanol $(300 \mathrm{~mL})$ for approximately $6-8 \mathrm{~h}$ and the filtered extract was concentrated in a rotary evaporator (Buchi) and stored in a refrigerator for further use.

\section{Formulation and optimization of nanosuspension}

Nanoprecipitation (bottom-up approach) was used for the formulation of the nanosuspensions [44], with some modifications. The plant extract was completely dissolved in ethanol, and the organic phase was slowly injected ( $1 \mathrm{~mL} / \mathrm{min}$ ) with a syringe connected to a thin Teflon tube into an aqueous phase containing the stabilizer 


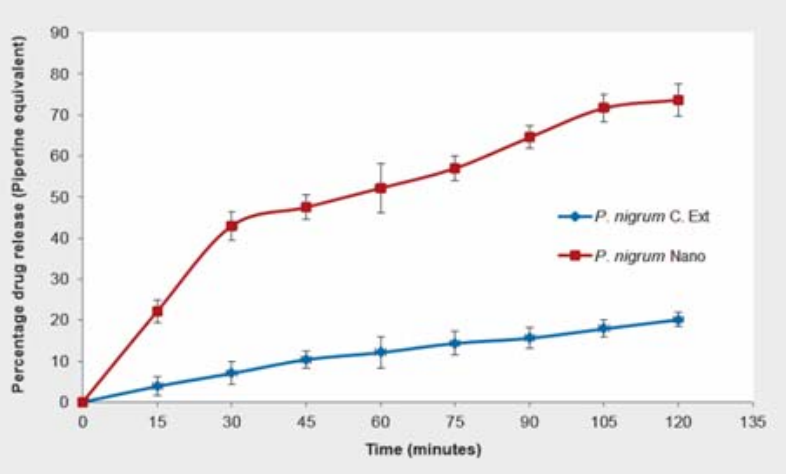

- Fig. 7 In vitro dissolution profile of $P$. nigrum nanosuspension and coarse extract. Results are expressed as the mean $\pm S D(n=3)$. C. Ext $=$ coarse extract, Nano $=$ nanosuspension.

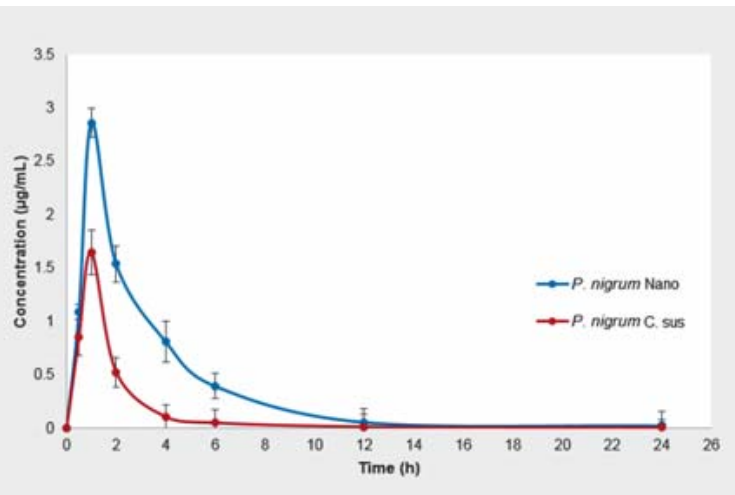

- Fig. 8 Concentration of piperine $(\mu \mathrm{g} / \mathrm{mL})$ in plasma samples of rats after oral administration of $P$. nigrum coarse suspension and nanosuspension. Results are expressed as the mean $\pm S D(n=3)$. C. sus $=$ coarse suspension, $\mathrm{Nano}=$ nanosuspension
(HPMC) with continuous stirring at $6000 \mathrm{rpm}$ for $6 \mathrm{~h}$ at room temperature. The requisite formulation parameters, i.e., amount of plant extract, concentration of stabilizer, and AS/S ratio, were optimized using the CCD of RSM. A standard stratagem of preliminary trials was used to determine the best possible conditions for the formulation of nanosuspensions. The CCD of RSM propounded 20 different conditions to perform the experiment for the optimal production of nanosuspensions by varying the amount of plant extract from 0.13 to $0.5 \%$, the concentration of the stabilizer from 0.25 to $2 \%$, and the AS/S ratio from 10 to 20 (Table 1S, Supporting information). The average particle size (zaverage; $\mathrm{nm}$ ) and PDI of the formulated nanosuspensions were selected as the response variables. The optimized nanosuspension (nanosuspension with a minimum particle size and appropriate PDI) was lyophilized at $-60^{\circ} \mathrm{C}$ for $72 \mathrm{~h}$. The freeze-dried sample was ground to a fine powder and used for solid-state characterization.

\section{Characterization of the nanosuspension}

The mean particle size (z-average; nm), PDI, and zeta potential of the nanosuspensions were measured by DLS using Malvern Zetasizer (Nano ZS). AFM (Shimadzu) was used for 3D characterization of the optimized nanosuspension. Surface morphology was evaluated by SEM (JEOL). Drug excipient interactions were evaluated by means of FTIR spectroscopy (Perkin Elmer). FTIR spectra of the crude herbal extract, optimized nanosuspension, and stabilizer (HPMC) were recorded.

\section{In vitro dissolution testing}

In vitro dissolution testing was conducted by adopting a modified version of the method used by Gera et al. [37]. USP dissolution apparatus type II (pharma test de) was used for dissolution testing of the coarse herbal extract and nanosuspension. For this purpose, an encapsulated 500-mg sample (coarse plant extract and lyophilized nanosuspension) was placed in $900 \mathrm{~mL}$ of the dissolution medium ( $0.1 \mathrm{M}$ phosphate buffer at $\mathrm{pH} 7.4)$ at a temperature of $37 \pm 0.5^{\circ} \mathrm{C}$ with a stirring rate of $50 \mathrm{rpm}$. Aliquots $(5 \mathrm{~mL})$ were withdrawn from the dissolution medium at predetermined time
- Table 3 Pharmacokinetic parameters after oral administration of P. nigrum nanosuspension and coarse suspension to experimental rats.

\begin{tabular}{|l|l|l|}
\hline Parameters & Nanosuspension & Coarse suspension \\
\hline $\mathrm{C}_{\max }(\mu \mathrm{g} / \mathrm{mL})$ & $2.86 \pm 0.24$ & $1.65 \pm 0.37$ \\
\hline $\mathrm{T}_{\max }(\mathrm{h})$ & $1.0 \pm 0.00$ & $1.0 \pm 0.00$ \\
\hline $\mathrm{AUC}_{0-24 \mathrm{~h}}(\mu \mathrm{gh} / \mathrm{mL})$ & $8.85 \pm 1.21$ & $3.28 \pm 0.86$ \\
\hline
\end{tabular}

Results are expressed as the mean $\pm S D(n=3)$. $C_{\max }=$ maximum concentration, $\mathrm{T}_{\max }=$ time to reach maximum concentration, $\mathrm{AUC}=$ area under the curve

intervals $(0,15,30,45,60,75,90$, and $120 \mathrm{~min})$, and the same volume of the prewarmed $\left(37^{\circ} \mathrm{C}\right)$ dissolution medium was immediately added to the dissolution vessel to maintain sink conditions. The concentration of the dissolved drugs (piperine equivalent) was spectrophotometrically determined at a wavelength of $342 \mathrm{~nm}$ ( $\lambda_{\max }$ of piperine). The concentration of piperine was evaluated from the regression equation generated from the calibration curve of standard piperine. The results are presented as drug dissolved (\%) for the coarse plant extract and nanosuspension. All experiments were conducted in triplicate, and results are presented as the mean $\pm \operatorname{SD}(n=3)$.

\section{In vivo pharmacokinetic study}

According to the International Ethical Guidelines and under the supervision of veterinary doctors of the Clinical Medicine and Surgery Department, UAF, the animal model was designed to conduct in vivo pharmacokinetic studies. The proposal was approved by the Synopsis Scrutiny Committee (No. Chem-354, dated 1502-2016) and endorsed by the Graduate Study Research Board through letter no. GDS/15501-4 dated 09-03-2016. Prof. Dr. Ghulam Muhammad oversaw the use of rats in this research; these rats were utilized as per the principles of the $3 R$ 's. For pharmacokinetic studies, male Wistar albino rats $(250 \pm 20 \mathrm{~g})$ were kept in an animal house for 1 week to acclimatize and were fed 
with a normal rat diet (rat chaw and grains). Two groups of three rats were made. Before the experiment, the rats were fasted overnight with free access to water. For comparison, the rats in the first group were orally administered the $P$. nigrum nanosuspension (50 mg/kg body weight), whereas those in the second group were orally administered the coarse suspension of $P$. nigrum at the same dose.

Blood samples $(0.5 \mathrm{~mL})$ were withdrawn by cardiac puncture in sodium heparinized tubes at predetermined time intervals of 0.5 , $1,2,4,6,12$, and $24 \mathrm{~h}$. Plasma was separated after centrifugation at $170 \times \mathrm{g}$ for $20 \mathrm{~min}$ and stored at $-20^{\circ} \mathrm{C}$ for further analysis. Piperine was extracted from the plasma samples by adopting the method used by Roy et al. [45] and quantified $(\mu \mathrm{g} / \mathrm{mL})$ by HPLC (model LC-10A; Shimadzu) using the standard calibration curve of piperine. A Supelco analytical HS (C-18) column having a length of $15 \mathrm{~cm}$, diameter of $4.6 \mathrm{~mm}$, and thickness of $5 \mu \mathrm{m}$ was used. The mobile phase (methanol:water, 70:30) flow rate was adjusted to $1 \mathrm{~mL} / \mathrm{min}$. The temperature of the column oven was set to $30^{\circ} \mathrm{C}$, and the pressure of the delivery pump was adjusted to 4413 kilopascal. A UV-visible detector (model SPD-10A; Shimad$\mathrm{zu}$ ) was set at a wavelength of $340 \mathrm{~nm}$. Acquisition software (class LC-10; Shimadzu) was used for analyzing the chromatograms.

\section{Determination of pharmacokinetic parameters}

Pharmacokinetic parameters such as peak plasma concentration $\left(C_{\max }\right)$ and time required to achieve peak plasma concentration $\left(T_{\max }\right)$ were determined directly from the concentration-time graph. The $A \cup C\left(A_{U} C_{0-24 h}\right)$ was determined by the trapezoidal method [37] using Microsoft Excel, 2007. Results are presented as the mean $\pm S D(n=3)$.

\section{Statistical analysis}

The CCD of RSM was used for optimizing the formulation parameters. The significance of the studied independent variables and their interactions were tested by ANOVA. Dissolution rates and values of various pharmacokinetic parameters are expressed as the mean $\pm \operatorname{SD}(n=3)$.

\section{Supporting information}

FTIR spectra of the $P$. nigrum coarse plant extract, $P$. nigrum nanosuspension, and stabilizer (HPMC), HPLC chromatograms of the $P$. nigrum coarse plant extract, $P$. nigrum nanosuspension (at $C_{\max }$ ) and piperine standard, and experimental conditions used in the optimization study are provided as Supporting Information.

\section{Acknowledgements}

The authors are grateful to the Higher Education Commission (HEC) of Pakistan for financial support [no. 20-2(3)/NIBGE, Fbd/ASIP/R\&D/HEC/ 2016/697] regarding the characterization studies. The authors would like to thank Enago (www.enago.com) for the English language review.

\section{Conflict of Interest}

The authors declare no conflict of interest

\section{References}

[1] Damanhouri ZA, Ahmad A. A Review on therapeutic potential of Piper nigrum L. (Black Pepper): The king of spices. Med Aromat Plants 2014; 3: $1-6$

[2] Chopra B, Dhingra AK, Kapoor RP, Prasad DN. Piperine and its various physicochemical and biological aspects: a review. Open Chem J 2016; 3: 75-96

[3] Taqvi SI, Shah AJ, Gilani AH. Blood pressure lowering and vasomodulator effects of piperine. J Cardiovasc Pharmacol 2008; 52: 452-458

[4] Agbor GA, Akinfiresoye L, Sortino J, Johnson R, Vinson JA. Piper species protect cardiac, hepatic and renal antioxidant status of atherogenic diet fed hamsters. Food Chem 2012; 134: 1354-1359

[5] Vijayakumar RS, Surya D, Senthilkumar R, Nalini N. Hypolipidemic effect of black pepper (Piper nigrum Linn.) in rats fed high fat diet. J Clin Biochem Nutr 2002; 32: 31-42

[6] Parganiha R, Verma S, Chandrakar S, Pal S, Sawarkar HA, Kashyap P. In vitro anti-asthmatic activity of fruit extract of Piper nigrum (Piperaceae). Inter J Herbal Drug Res 2011; 1: 15-18

[7] Nirwane AM, Bapat AR. Effect of methanolic extract of Piper nigrum fruits in ethanol-CCl4 induced hepatotoxicity in Wistar rats. Der Pharmacia Lettre 2012; 4: 795-802

[8] Ganesh P, Kumar RS, Saranraj P. Phytochemical analysis and antibacterial activity of pepper (Piper nigrum L.) against some human pathogens. Cent Eur J Expl Biol 2014; 3: 36-41

[9] Srinivasan K. Black pepper and its pungent principle-piperine: a review of diverse physiological effects. Crit Rev Food Sci Nutr 2007; 47: 735-748

[10] Dudhatra GB, Mody SK, Awale MM, Patel HB, Modi CM, Kumar A, Kamani DR, Chauhan BN. A comprehensive review on pharmacotherapeutics of herbal bioenhancers. Sci World J 2012; 2012: 1-33

[11] Mittal R, Gupta RL. In vitro antioxidant activity of piperine. Exp Clin Pharmacol 2000; 22: 271-274

[12] Gorgani L, Mohammadi M, Najafpour GD, Nikzad M. Piperine - the bioactive compound of black pepper: from isolation to medicinal formulations. Compr Rev Food Sci Food Saf 2017; 16: 124-140

[13] Li M, Azad M, Davé R, Bilgili E. Nanomilling of drugs for bioavailability enhancement: a holistic formulation-process perspective. Pharmaceutics 2016; 8: 1-35

[14] Lakshmi P, Kumar GA. Nanosuspension technology: A review. Int J Pharm Pharm Sci 2010; 2: 35-40

[15] Bhadoriya SS, Mangal A, Madoriya N, Dixit P. Bioavailability and bioactivity enhancement of herbal drugs by "Nanotechnology": a review. J Ceram Process Res 2011; 8: 1-7

[16] Attari Z, Bhandari A, Jagadish P, Lewis S. Enhanced ex vivo intestinal absorption of olmesartan medoxomil nanosuspension: Preparation by combinative technology. Saudi Pharm J 2016; 24: 57-63

[17] Huang S, Chang WH. Advantages of nanotechnology-based chinese herb drugs on biological activities. Curr Drug Metab 2009; 10: 905-913

[18] Pawar SS, Dahifale BR, Nagargoje SP, Shendge RS. Nanosuspension technologies for delivery of drugs. J Nanosci Nanotechnol 2017; 4: 59-66

[19] Sutradhar KB, Khatun S, Luna IP. Increasing possibilities of nanosuspension. J Nanotechnol 2013; 2013: 1-12

[20] Garai D, Kumar V. Response surface optimization for xylanase with high volumetric productivity by indigenous alkali tolerant aspergillus candidus under submerged cultivation. 3 Biotech 2013; 3: 127-136

[21] Bezerra MA, Santelli RE, Oliveira EP, Villar LS, Escaleira LA. Response surface methodology (RSM) as a tool for optimization in analytical chemistry. Talanta 2008; 76: 965-977

[22] Lee KM, Hamid SBA. simple response surface methodology: investigation on advance photocatalytic oxidation of 4-chlorophenoxyacetic acid using UV-active ZnO photocatalyst. Materials (Basel) 2015; 8: 339-354 
[23] Selvam K, Sudhakar C, Govarthanan M, Thiyagarajan P, Sengottaiyan A, Senthilkumar B, Selvankumar T. Eco-friendly biosynthesis and characterization of silver nanoparticles using Tinospora cordifolia (Thunb.) Miers and evaluate its antibacterial, antioxidant potential. J Radiat Res Appl Sci 2017; 10: 6-12

[24] Matteucci ME, Hotze MA, Johnston KP, Williams RO. Drug nanoparticles by antisolvent precipitation: mixing energy versus surfactant stabilization. Langmuir 2006; 22: 8951-8959

[25] Dalvi SV, Dave RN. Controlling particle size of a poorly water-soluble drug using ultrasound and stabilizers in antisolvent precipitation. Ind Eng Chem Res 2009; 48: 7581-7593

[26] Hao J, Gao Y, Zhao J, Zhang J, Li Q, Zhao Z, Liu J. Preparation and optimization of resveratrol nanosuspensions by antisolvent precipitation using box-behnken design. AAPS PharmSciTech 2015; 16: 118-128

[27] Thorat AA, Dalvi SV. Liquid antisolvent precipitation and stabilization of nanoparticles of poorly water soluble drugs in aqueous suspensions: Recent developments and future perspective. Chem Eng J 2012; 181: $1-34$

[28] Ahuja BK, Jena SK, Paidi SK, Bagri S, Suresh S. Formulation, optimization and in vitro-in vivo evaluation of febuxostat nanosuspension. Int ] Pharm 2015; 478: 540-552

[29] Fritzen-Garcia MB, Zanetti-Ramos BG, Oliveira CSD, Soldi V, Pasa AA, Creczynski-Pasa TB. Atomic force microscopy imaging of polyurethane nanoparticles onto different solid substrates. Mater Sci Eng C 2009; 29: 405-409

[30] Zanetti-Ramos BG, Fritzen-Garcia MB, Creczynski-Pasa TB, Oliveira CSD, Pasa AA, Soldi V, Borsali R. Characterization of polymeric particles with electron microscopy, dynamic light scattering, and atomic force microscopy. Part Sci Technol 2010; 28: 472-484

[31] Papdiwal AP, Pande VV, Aher S. Investigation of effect of different stabilizers on formulation of zaltoprofen nanosuspension. Int J Pharm Sci Rev Res 2014; 27: 244-249

[32] Mishra B, Sahoo J, Dixit PK. Formulation and process optimization of naproxen nanosuspensions stabilized by hydroxy propyl methyl cellulose. Carbohydr Polym 2015; 127: 300-308

[33] Shi SC, Wu JY, Huang TF. Raman, FTIR, and XRD study of MoS2 enhanced hydroxypropyl methylcellulose green lubricant. Opt Quant Electron 2016; 48: 1-9
[34] Nath B, Roy TK. Thermal characterization and screening of formulation variables of atorvastatin calcium immediate release tablet. JSM Nanotechnol Nanomed 2017; 5: 1-8

[35] Motawie A, Ahmed S, El-Sabbagh E, Mansour N, Abulyazied D, Ali E. Physicomechanical properties of nano polystyrene nanocomposites. Egypt J Chem 2017; 60: 261-276

[36] Wang Y, Zhang D, Liu Z, Liu G, Duan C, Jia L, Feng F, Zhang X, Shi Y, Zhang Q. In vitro and in vivo evaluation of silybin nanosuspensions for oral and intravenous delivery. Nanotechnol 2010; 21: 1-12

[37] Gera S, Talluri S, Rangaraj N, Sampathi S. Formulation and evaluation of naringenin nanosuspensions for bioavailability enhancement. AAPS PharmSciTech 2017; 18: 3151-3162

[38] Kakran M, Sahoo G, Li L. Fabrication of nanoparticles of silymarin, hesperetin and glibenclamide by evaporative precipitation of nanosuspension for fast dissolution. Pharm Anal Acta 2015; 6: 1-7

[39] Tian X, Li H, Zhang D, Liu G, Jia L, Zheng D, Shen J, Shen Y, Zhang Q. Nanosuspension for parenteral delivery of a p-terphenyl derivative: preparation, characteristics and pharmacokinetic studies. Colloids Surf B Biointerfaces 2013; 108: 29-33

[40] Borhade V, Pathak S, Sharma S, Patravale V. Formulation and characterization of atovaquone nanosuspension for improved oral delivery in the treatment of malaria. Nanomed 2014; 9: 649-666

[41] Rao YM, Kumar PM, Apte S. Formulation of nanosuspensions of albendazole for oral administration. Current Nanosci 2008; 4: 53-58

[42] Ravichandran R. Nanotechnology-based drug delivery systems. NanoBiotechnol 2009; 5: 17-33

[43] Abhinav M, Neha J, Anne G, Bharti V. Role of novel drug delivery systems in bioavailability enhancement: at a glance. Int J Drug Deliv Technol 2016; 6: 7-26

[44] Thadkala K, Nanam PK, Rambabu B, Sailu C, Aukunuru J. Preparation and characterization of amorphous ezetimibe nanosuspensions intended for enhancement of oral bioavailability. Int J Pharm Investig 2014; 4: 131 137

[45] Roy S, Gupta A, Chopra H, Maurya AK, Tripathi S, Sahu RK, Tomar H, Sharma SC. Pharmacokinetic study of piperine in mice plasma after orally and intravenous administration. Int J Drug Deliv 2012; 4: 107-112 